\section{Neue Bücher}

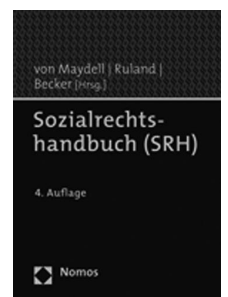

Bernd Baron von

Maydell, Franz

Ruland, Ulrich

Becker (Hg.): Sozi-

alrechtshandbuch

(SRH). 4. Auflage.

Nomos Verlagsgesellschaft, $\mathrm{Ba}$ -

den-Baden 2008. 1.502 Seiten. 148,- Euro. ISBN 978-3-8329-2.

In der vierten Auflage des »Sozialrechtshandbuchs « findet sich auf eineinhalbtausend Seiten eine aktuelle Gesamtdarstellung des deutschen Sozialrechts. Markenzeichen des Werkes ist es, dass für die einzelnen Kapitel die jeweils bundesweit anerkannten Experten verantwortlich zeichnen. Erfreulicherweise beschränkt sich die Darstellung nicht auf das Recht. Ausführlich werden historische und ökonomische Grundlagen der Sozialen Sicherung dargestellt und auch ein Blick in die Zukunft des Sozialstaats gewagt. Für Untergangsszenarien sieht der renommierte Richard Hauser hier keinen Anlass. Die Beziehungen zwischen Leistungsberechtigten und Leistungsverwaltung, Fragen des Verwaltungsverfahrens und des Datenschutzes werden ebenso kompetent abgehandelt wie die einzelnen Felder der Sozialleistungen. Das besondere Verdienst dieser Darstellungen liegt darin, dass nicht nur der Jurist zuverlässig den aktuellen Stand von Rechtsprechung und Gesetzgebung vorfindet, sondern vor allem der Nichtjurist die Systemlogiken und Grundanliegen der einzelnen Leistungsbereiche allgemeinverständlich nachlesen kann. Die Darstellungen verlieren sich nicht in Details, sondern stellen die Trends heraus, ohne aber wichtige Einzelheiten zu vernachlässigen oder gar zu verfälschen. Das Sozialrechtshandbuch ist gleichzeitig Studienbuch und Nachschlagewerk für alle, die mit den rechtlichen und politischen Rahmenbedingungen der Sozialen Arbeit zu tun haben.

Werner Hesse

Werner Hesse ist Geschäftsführer für Personal und Recht beim Paritätischen Wohlfahrtsverband-Gesamtverband $e . V$. E-Mail sozialrecht@paritaet.org

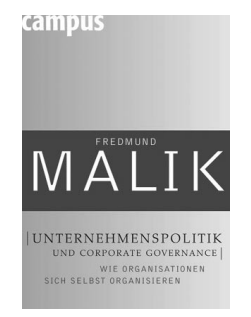

Fredmund Malik: Unternehmenspolitik und Corporate Governance - wie Organisationen sich selbst organisieren. Campus Verlag, Frankfurt

am Main 2008. 363 Seiten. 39,90 Euro. ISBN 978-3-59338286-9.

Maliks neues Werk macht den Versuch durch Anwendung der Kybernetik auf Management, dem Problem der Hyperkomplexität von Systemen so zu begegnen, dass Manager (wieder) Steuerungs- und Regelungsfunktionen in ihren Unternehmen wahrnehmen können. Dabei unterstellt er, dass komplexe Systeme prinzipiell undurchschaubar und unberechenbar sind, wenngleich er aufgrund seiner jahrzehntelangen Managementerfahrung dennoch den Versuch unternimmt, die Gesetzmäßigkeiten komplexer Systeme dem Leser verstehbar zu machen. Er lädt ihn ein, mit ihm auf die Reise zu einem anderen Weltbild und damit einhergehend letztlich zu einem neuen Handlungskonzept zu gehen. Maliks Adressaten sind die Top-Manager in Wirtschaftsunternehmen. Und obwohl gleich zu Beginn auch Krankenhäuser als Unternehmen explizit benannt werden, so stellt sich doch die Frage, ob sich von diesem Buch die breite Masse sozialwirtschaftlicher Unternehmen angesprochen fühlen wird, sagen wir einmal eine Schuldnerberatungsstelle oder ein Frauenhaus. Die Mehrzahl der Führungskräfte in der Sozialwirtschaft wird deshalb ab der Seite 35 möglicherweise nicht mehr interessiert weiterlesen wollen - was jedoch schade wäre: und zwar nicht weil Malik die Leitungsverantwortlichen in einer Sozialstation oder einer Schwangerenberatungsstelle eher nicht zu den Top-Managern von Wirtschaftsunternehmen zählt, sondern weil sich Sozialeinrichtungen und deren Verbände mit ihren Kodizes des Unternehmenshandelns längst als wichtige Elemente komplexer Systeme verstehen. Durch die Implementation von Wissensmanagement zum Beispiel reagieren sie bereits auf die von Malik prognostizierten »Veränderungen von einem Sachwissen hin zu einem Systemwissen«. Es soll keineswegs despektierlich wirken oder die Bedeutung von Maliks neuem Buch schmälern: Viele Aussagen des in der St. Gallener Management-Tradition stehenden Fredmund Malik werden Menschen in Leitungsverantwortung sozialer Unternehmen nicht mehr so völlig neu vorkommen, weil deren Spitzenverbände seit langem Führungsseminare zum neuen St. Gallener Managementmodell in der Schweiz anbieten (ehemals Diakonische Akademie Deutschland) oder auch eigene Führungsakademien aufbauen, die allesamt das Ziel haben, das »Agieren in Komplexität « zu erleichtern, Managementmodelle anzubieten, Anleitungen zur Selbstorganisation zu geben. Der in der Sozialwirtschaft agierende Leser wird Maliks Thesen mal mehr und mal weniger zustimmen können: Bei dem häufig anzutreffenden Vollzugsdefizit einer sich permanent ändernden Sozialgesetzgebung wird er bei der These, dass Regierungspolitik in ihrer bisherigen Form mehr »Quelle von Störungen « ist, sehr nahe bei Malik sein. Das trifft auch auf die These einer notwendigen Befähigung der Unternehmen zur Selbstorganisation und Selbstbestimmung zu. Doch Zweifel an der Umsetzbarkeit von Maliks Ideen kommen all denjenigen von uns, die in unserem überregulierten sozialen Dienstleistungssystemen eher Elemente der Planwirtschaft als das Ideal »freier Märkte« - als Maliks Referenzmodel - haben. Natürlich wurden bisher den Leitungsverantwortlichen in sozialen Unternehmen in ihrer Ausbildung oft keine Lehrinhalte über komplexe, sich selbst organisierende Systeme vermittelt. Aber man fragt sich sehr schnell auch: »Was hätte die Leitung einer Schuldnerberatungsstelle denn mit diesem Wissen bewirken können? « Wohlgemerkt, dies kann und darf keine Kritik an Malik sein, sondern eher an den Rahmenbedingungen und den Gesetzgebungsprozessen im Sozialbereich: Wenn also zwei der vier »Sub-Controls« (Strategie, Führungskräfte, Struktur, Kultur) als »staatliche Dienstleistungsstruktur « und »Kultur des Sparens« bereits staatlich vorgegeben sind, so können diese eingebettet in die Maliks Master Controls Umwelt, Unternehmenspolitik, Corporate Governance gewiss nur teilweise ihre Wirkungen entfal- 
ten. Der sozialstaatliche Perfektionist wird die Adaption dieses Modells eher kritisch beurteilen, der Optimist vertraut zumindest auf die Wirkung eines »halbvollen Glases « in der Hoffnung, dass zukünftig bei einer Deregulierung des Sozialbereichs durch die Politik, auch diejenigen mitgestalten und lenken, die am meisten von den sozialen Interessen der Kunden verstehen: die Dienstleistungserbringer der Sozialwirtschaft selbst. Wenn nach Malik der ständige Wandel die einzige Konstante bei der Veränderung komplexer Systeme ist, darf man auch hier die Hoffnung nicht aufgeben.

Uwe Schwarzer

Uwe Schwarzer ist beim Vorstand des Diakonischen Werkes der EKD zuständig für Fragen des Qualitätsmanagements, des Verbraucherschutzes und für innovative Sozialprojekte. E-Mailschwarzer@diakonie.de

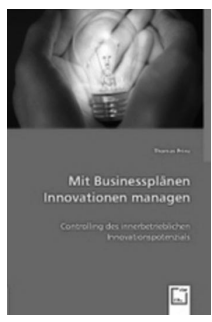

Thomas Prinz: Mit BusinessPlänen Innovationen managen. VDM Verlag Dr. Müller, Saarbrücken 2008. 326 Seiten. 79,Euro. ISBN 978-3-8364-9182-2.

Innovationen sind in den Augen des Autors notwendig zum Erhalt und Ausbau der Wettbewerbsfähigkeit eines Unternehmens, auch im Sozial- und Verwaltungsbereich. Einerseits geht es um das Heben von innovativem Potenzial im Betrieb, andererseits um die Begrenzung des Risikos bei der Entwicklung von innovativen Projekten oder Abläufen. Eine Gradwanderung, die durch das flexible Nutzen von Instrumenten im Rahmen eines Businessplans erleichtert werden soll. Im Zentrum steht der interne Businessplan, in den abhängig von den jeweiligen Anforderungen eine Reihe von Instrumenten wie die Nutzwertanalyse oder Wirtschaftlichkeitsrechnung integriert werden kann. Der Businessplan wird dabei als ein modularer Rahmen gesehen, der mit Instrumenten in Abhängigkeit von der Zielsetzung und den Rahmenbedingungen befüllt werden muss. Vor diesem Hintergrund erfolgt eine Expertenbefragung, um Hinweise zur Gestaltung des InnovationsControllings zu erhalten. Anschließend werden Erfolgsfaktoren aus der Analyse von gescheiterten Unternehmern zusammengetragen und Kriterien für die Bewertung eines internen Businessplans entwickelt. Insgesamt bietet dieser Ansatz des modularen und dynamischen Innovationscontrollings eine gute Basis, um mit Hilfe von internen Businessplänen auch für ältere, vielleicht weniger innovationsfreudige Organisationen einen Weg zur Erneuerung zu ermöglichen, der das natürlicherweise innewohnende Risiko von Innovationen durch möglichst rationale Entscheidungshilfen begrenzen hilft. Der Autor Thomas Prinz war mehrere Jahre Leiter einer Konzernrevision, strategischer Controller und Unternehmensberater. Seit 2007 hat er eine Professur für Controlling, Finanzmanagement und Unternehmensplanung an der FH Oberösterreich Campus Linz in den Studiengängen Sozial- und Verwaltungsmanagement.

\section{Paul Brandl}

Prof. (FH) Dr. Paul Brandl lehrt an der Fachhochschule Oberösterreich am Campus Linz im Studiengang Sozialmanagement.E-Mail paul.brandl@fh-linz.at

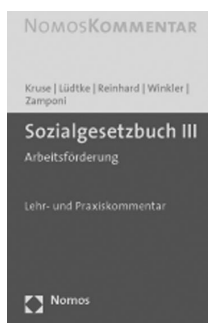

Jürgen Kruse, Peter-Bernd Lüdtke, Hans-Joachim Reinhard, Jürgen Winkler, Irene Zamponi: Sozialgesetzbuch III. Arbeitsförderung. Lehr- und Praxiskommentar. Nomos Verlagsgesellschaft BadenBaden 2008. 1.060 Seiten 79,Euro. ISBN 978-3-8329-0309-1.

Der neue Kommentar zur Arbeitsförderung verfolgt den besonderen Anspruch, die komplexe Gesetzesmaterie des SGB III durch einen geschichtlichen Überblick zur Entstehung und Weiterentwicklung des Sozialgesetzbuchs wie auch eine Einführung zur Entstehungsgeschichte der jeweiligen Einzelvorschriften verständlicher zu machen. Das ist zum Verständnis einzelner Paragrafen nützlich; für eine umfassende Deutung der aktuellen Gesetzgebung fehlt jedoch ein wenig der Blick auf rechtkreisübergreifende Diskussionen in der Arbeitsmarktpolitik. Für Praktiker in den Einrichtungen und Diensten der Sozialen Arbeit, etwa in Arbeitslosenberatungsstellen oder Beschäftigungsinitiativen, dürfte der Nomos-Kommentar je nach Autorenbeitrag

unterschiedlich gut lesbar sein: In den Abschnitten zur Erläuterung spezieller Regelungen für die Teilhabe behinderter Menschen am Arbeitsleben leistet Irene Zamponi gute Übersetzungsarbeit. Demgegenüber dürften die von Bundesrichter Lüdtke i. R. erstellten Passagen etwa zu den Anspruchsvoraussetzungen für das Arbeitslosengeld I eher von Nutzen für Anwälte und Richter sein. Leider fehlt es dem Kommentar zum Teil an aktuellem Praxiswissen in der Arbeitsmarktförderung und Sozialen Arbeit, etwa wenn die Eingliederungsvereinbarungen nach dem SGB III als vergleichbar mit den Hilfeplänen der Jugendhilfe (SGB VIII) dargestellt werden. Fazit: Angesichts der bevorstehenden Gesetzgebung wird der neue SGB III-Kommentar nur für kurze Zeit auf aktuellem Stand sein. Meines Erachtens nutzt er eher Anwälten und Richtern als Praktikern in der Sozialen Arbeit. Die sollten den »Leitfaden für Arbeitslose des Fachhochschulverlages kennen.

Tina Hofmann

Tina Hofmann ist Referentin für Jugendsozialarbeit im Paritätischen Wohlfahrtsverband-Gesamtverband e. V. E-Mail jugendsozialarbeit@paritaet.org 